\title{
Controlling motion artefact levels in MR images by suspending data acquisition during periods of head motion
}

\author{
Rémi Castella1* (D) | Lionel Arn1,2* | Estelle Dupuis ${ }^{1}$ | Martina F. Callaghan ${ }^{3}$ | \\ Bogdan Draganski ${ }^{1,4}$ | Antoine Lutti ${ }^{1}$ \\ ${ }^{1}$ LREN, Department for Clinical Neurosciences, CHUV, Lausanne, Switzerland \\ ${ }^{2}$ Ecole Polytechnique Fédérale de Lausanne, Lausanne, Switzerland \\ ${ }^{3}$ Wellcome Trust Centre for Neuroimaging, UCL Institute of Neurology, University College London, London, United Kingdom \\ ${ }^{4}$ Max Planck Institute for Human Cognitive and Brain Sciences, Leipzig, Germany
}

Correspondence Antoine Lutti, Chemin de Mont-Paisible 16, 1011 Lausanne, Switzerland.

Email: antoine.lutti@chuv.ch

\section{Funding information}

Supported by the Roger De Spoelberch Foundation and the Partridge Foundation.

A.L. is supported by the Roger De

Spoelberch Foundation. B.D. is supported

by the Swiss National Science

Foundation (NCCR Synapsy, project grant 32003B_159780), Foundation

Parkinson Switzerland, and Foundation

Synapsis. The research leading to these results has received funding from the European Union's Horizon 2020

Research and Innovation Program under grant agreement 720270 (HBP SGA1)
Purpose: Head movements are a major source of MRI artefacts. Prospective motion correction techniques significantly improve data quality, but strong motion artefacts may remain in the data. We introduce a framework to suspend data acquisition during periods of head motion over a predefined threshold.

Methods: Data was acquired with prospective motion correction and an external optical tracking system. A predictor of motion impact was introduced that accounts for the amplitude of the signal acquired at the time of the motion. From this predictor, a threshold was defined to trigger the suspension of data acquisition during periods of motion. The framework was tested on 5 subjects, 2 motion behaviors, and 2 head coils (20 and 64 channels).

Results: The best improvements in data quality were obtained for a threshold value of 0 , equivalent to suspending the acquisition based on head speed alone, at the cost of a long prolongation of scan time. For threshold values $\sim 3.5 \mathrm{e}^{-4}$, image quality was largely preserved, and prolongation of scan time was minimal. Artefacts occasionally remained with the 64-channel head coil for all threshold values, seemingly due to head movement in the sharp sensitivity profile of this coil.

Conclusion: The proposed suspension strategy is more efficient than relying on head speed alone. The threshold for suspension of data acquisition governs the tradeoff between image degradation due to motion and prolonged scan time, and can be tuned by the user according to the desired image quality and participant's tolerability.

\section{KE Y W O R D S}

acquisition suspension, head movement, MRI artefacts, multi-parameter mapping, prospective motion correction, quantitative MRI

\footnotetext{
*These authors contributed equally to this work.
}

This is an open access article under the terms of the Creative Commons Attribution-NonCommercial License, which permits use, distribution and reproduction in any medium, provided the original work is properly cited and is not used for commercial purposes.

(C) 2018 The Authors Magnetic Resonance in Medicine published by Wiley Periodicals, Inc. on behalf of International Society for Magnetic Resonance in Medicine 


\section{1 | INTRODUCTION}

Head movement leads to severe artefacts in MR images that hamper the diagnostic assessment of brain MRI scans ${ }^{1}$ and have a strong impact on morphological, ${ }^{2}$ diffusion-based, ${ }^{3}$ and functional ${ }^{4}$ measures of the brain. Quite naturally, the effects of head movement are biased toward noncompliant populations, which limits the use of MRI for the study of brain pathology, an essential cornerstone of clinical neuroscience.

Motion correction can be implemented using prospective or retrospective methods and using passive or active markers of head position. ${ }^{5}$ Prospective motion correction (PMC) techniques act in real time during image acquisition by adjusting the MRI scanner to account for patient movements. ${ }^{6}$ With PMC methods, the acquired MRI data is already corrected for motion effects, avoiding the need for dedicated processing steps. ${ }^{7.8}$ In this study, we use the PMC method introduced by Zaitsev et al., ${ }^{6}$ based on external optical measures of brain motion using the tracking system introduced by Maclaren et al. ${ }^{9}$ (KinetiCor, HI, Honolulu). This technique may be implemented with minimal changes to pulse sequences but requires specially dedicated software libraries to integrate the motion information into the software environment of the MRI scanner. A functional MRI study using this system has reported improvements in SNR by $30 \%$ to $40 \%$ and an increase in the numbers of significantly activated voxels by $70 \%$ to $330 \% .{ }^{4}$ Improvements in the precision of relaxometry measures in the range of $11 \%$ to $24 \%$ have also been reported using this PMC technique. ${ }^{10}$

The lag time between tracking of the marker position and acquisition update $\left(32 \mathrm{~ms}^{6}\right)$ can induce erroneous motion correction for fast motion, imposing an upper limit on head velocities that can be accurately corrected. ${ }^{6,7}$ To overcome this limitation, we introduce a framework that allows the interruption of data acquisition during periods of head motion detrimental to image quality. Data acquisition is resumed automatically by the MRI scanner following motion periods. A similar strategy has been introduced recently to allow reacquisition of data for excessive head motions ${ }^{6,11-14}$ or motion artefacts. ${ }^{15,16}$ Here, we base our strategy on a predictor of the impact of head motion on image quality, which accounts for the amplitude of the signal acquired at the time of the motion as well as head speed. This predictor allows suspension of data acquisition with optimal efficiency by continuing the sampling process during periods of strong motion when the amplitude of the acquired signal is low. Importantly, the presented framework allows the user to set the maximal amount of image degradation deemed acceptable in the MRI data. This determines the threshold for triggering of the suspension of data acquisition and governs the tradeoff between the quality of the MR images and the extension of the scan duration due to the suspension. This value can be adjusted on a studyspecific basis depending on the compliance of the population of interest and the desired level of image quality.

\section{2 | METHODS}

\section{1 | Predictor of motion impact}

To obtain a predictor of image degradation from motion trajectories, we follow the framework introduced by Todd et al. ${ }^{4}$ They highlighted the importance of the amplitude of the signal acquired at the time of the motion in developing aggregate measures of motion impact over the whole duration of the acquisition. The encoding-weighted integrated motion metric (Mew) is defined as:

$$
M e w=\sum_{i=1}^{N} \operatorname{Mew}_{i}=\sum_{i=1}^{N} S_{i} * W_{i}
$$

where $S_{i}$ represents the head speed at the time of the acquisition of the $i$ th $\mathrm{k}$-space line and $W_{i}$ is the weight of the $i$ th $\mathrm{k}$ space line to Mew-the aggregate measure of motion impact over the whole 3D acquisition. For the FLASH acquisition used here, the total number of acquired lines $N$ is the product of the number of phase-encode steps in the phase and partition (secondary phase-encoded) directions.

In line with Ref. 4 , we take $\mathrm{W}_{\mathrm{i}}$ as the norm of the $i$ th acquired line, calculated by taking the Fourier transform of the magnitude image and summed over all readout points. Figure 1A shows an example set of weights that show high-/ low-frequency variations due to the inner(partition)/outer (phase) phase-encoded directions, sampled using a Cartesian trajectory. $S_{i}$ is calculated as in Ref. 4 :

$$
S_{i}=\sqrt{v_{i}{ }^{2}+\omega_{i}{ }^{2}},
$$

where $v_{i}$ and $\omega_{i}$ are the translation and rotation velocities of the center of the FOV of the encoding box (in $\mathrm{mm} / \mathrm{s}$ and $\mathrm{deg} / \mathrm{s}$ ), computed from the optical camera data as the difference in position and orientation between the $(i-1)$ th and $i$ th timeframes divided by the corresponding time interval.

Figure 1C shows the time course of $\mathrm{Mew}_{\mathrm{i}}$ calculated from the weights and head speeds of Figures 1A and 1B. Because the weights vary by several orders of magnitude throughout the acquisition, a given head speed may lead to values of $\mathrm{Mew}_{\mathrm{i}}$, which may vary by the same amount depending on the time of occurrence of head motion. This forbids the use of a unique threshold value of Mew for the whole acquisition to detect head motion and trigger the suspension of the acquisition. Instead, this threshold value should be defined by considering deviations of $\mathrm{Mew}_{\mathrm{i}}$ $\left(\Delta \mathrm{Mew}_{\mathrm{i}}\right)$ from its values when no significant head motion is present:

$$
\Delta \text { Mew }_{i}=\text { Mew }_{i}-\text { Mew }_{\text {compliant }, i} .
$$

Here, Mew compliant, $\mathrm{i}_{\mathrm{i}}$ is the value of $\mathrm{Mew}_{\mathrm{i}}$ in compliant populations. Significant motion can then be identified in real time as yielding values of $\Delta \mathrm{Mew}_{\mathrm{i}}$ above 0 , regardless of the occurrence of the motion in k-space (see Figure 1D). 

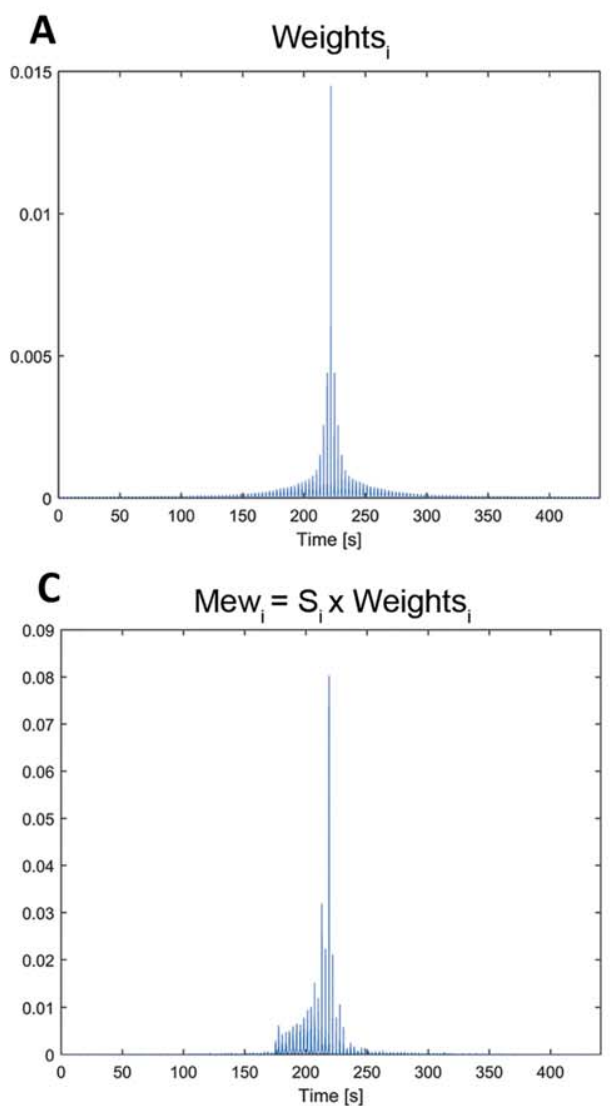
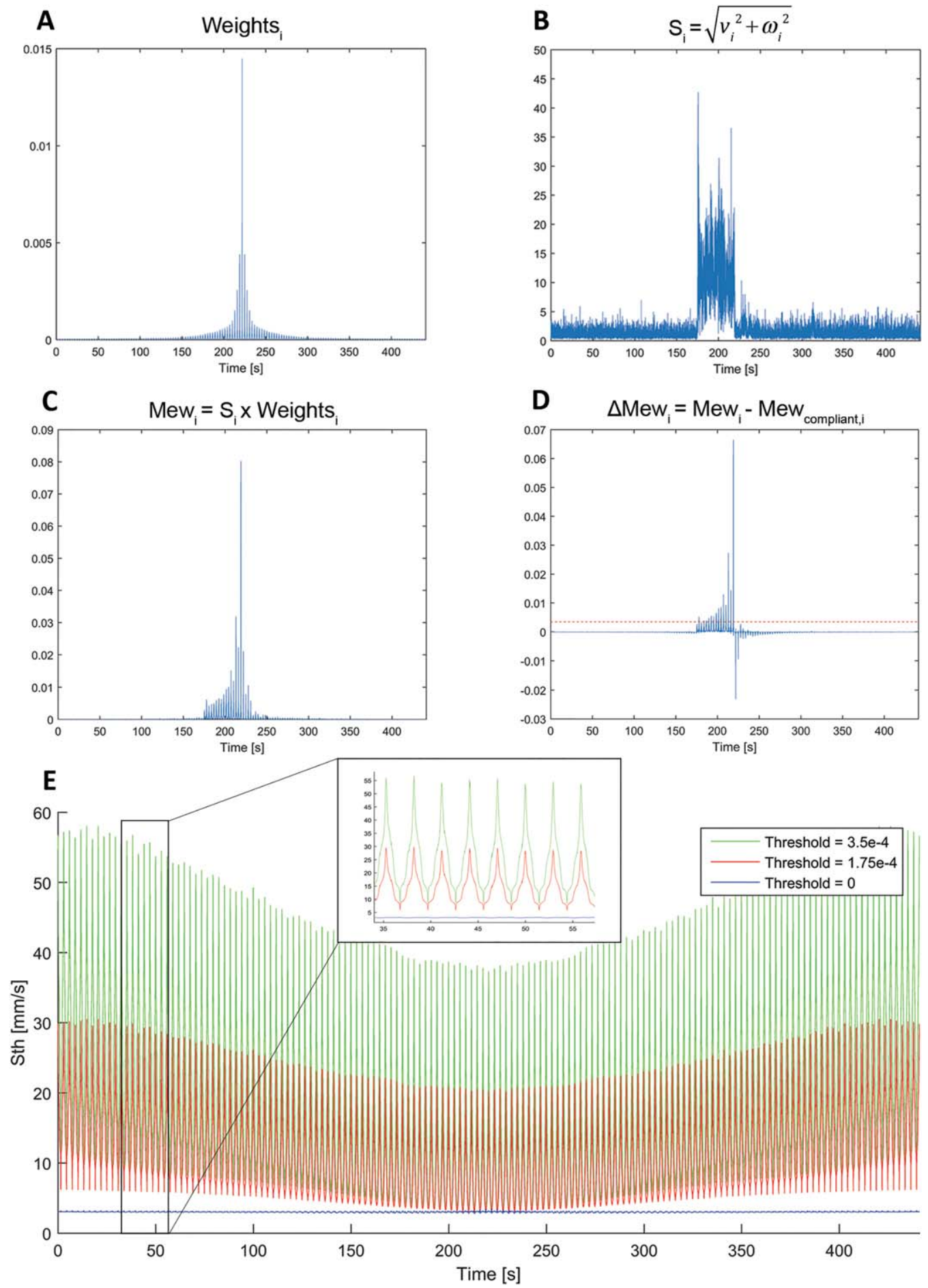

F I G URE 1 (A) Example time evolution of the amplitude of the acquired signal (weights) for the double phase-encoded Cartesian sampling used in

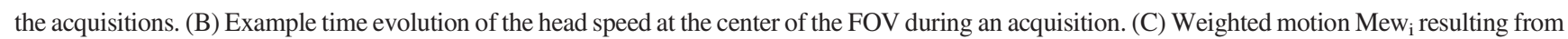
the point-wise multiplication of A and B. (D) Time evolution of $\Delta$ Mewi, the difference between Mewi and reference values obtained from a compliant population. Values of $\Delta \mathrm{Mew}_{\mathrm{i}}>0$ highlight time points where significant head motion took place. The red dashed line is an example choice of value for $\Delta \mathrm{Mew}_{\mathrm{th}}$, the threshold $\Delta \mathrm{Mew}$ value used to suspend data acquisition. (E) Time evolution of the threshold head speed value used for the suspension of data acquisition, equivalent to a constant $\Delta \mathrm{Mew}_{\text {th }}$ for all the acquisition (red dashed lines in $\mathrm{D}$ ).

$\Delta \mathrm{Mew}_{\mathrm{i}}$, deviations of $\mathrm{Mew}_{\mathrm{i}} ; \Delta \mathrm{Mew}_{\mathrm{th}}$, threshold of $\Delta \mathrm{Mewi}$; Mewi, encoding-weighted integrated motion metric

Our definition of $\mathrm{Mew}_{\text {compliant,i }}$ should account for all possible values of $S_{i}$ due to noise in the estimation of the marker position/orientation and head motion of physiological origin (e.g., heartbeat and respiration). The former represents an intrinsic limit of the PMC system (typically a few microns ${ }^{9}$ ), and the latter can be accurately corrected by the 
PMC system and does not need to be addressed here. Therefore, $\mathrm{Mew}_{\text {compliant,i }}$ was computed from:

1. A set of reference weights obtained from PDw and $T_{1} w$ images acquired in 10 compliant participants (same acquisition sequence and image resolution as the current study) to account for the variability in weights across a population. These 10 participants ( 5 females and 5 males, age mean/SD: 37.5/13.8) were independent from those involved in the motion experiments.

2. Samples of $S$ obtained from 3 motion time courses of 5 compliant participants. A histogram of these samples of $S$ is shown in Supporting Information Figure S1.

Multiple sample values of $\mathrm{Mew}_{\text {compliant,i }}$ were obtained by combination of the reference weights with all possible permutations of the $\mathrm{S}$ samples. The reference $\mathrm{Mew}_{\text {compliant,i val- }}$ ues were obtained by taking the mean across the

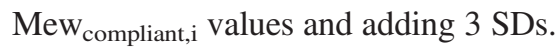

During image acquisition, the instantaneous value of $\mathrm{Mew}_{\mathrm{i}}$ was calculated from the product of the instantaneous head speed with the corresponding weight, calculated by taking the mean across the reference weights. These mean reference weights, calculated from an independent dataset as described above, were used for all study participants and motion experiments. Periods of motion are identified in real time as those yielding values of $\Delta \mathrm{Mew}_{\mathrm{i}}$ above 0 (see Figure 1D). From these values, an aggregate measure of motion degradation over the entire acquisition can be calculated using:

$$
\overline{\Delta M e w}=\sum_{i=1}^{N} \Delta M e w_{i}>0 .
$$

The aggregate measure of motion degradation $\overline{\Delta M e w}$ is the sum of all the values of $\Delta \mathrm{Mew}_{\mathrm{i}}$ above 0 encountered during the acquisition.

\section{2 | Real-time suspension of data acquisition during periods of motion}

In the first part of this study, we show the relationship between the quality of the acquired data ( $\mathrm{SD}$ of $\mathrm{R}_{2}^{*}\left[\mathrm{SDR}_{2}^{*}\right]$ : see marker of image degradation section below) and $\overline{\Delta \mathrm{Mew}}$. This relationship establishes $\overline{\Delta \mathrm{Mew}}$ as a predictor of the impact of head motion on image quality. In the second part of this study, we use this predictor to monitor in real time during data acquisition the impact of head motion on data quality and pause the acquisition when the predicted degradation exceeds a predetermined threshold.

Although in principle the quality of an MRI image cannot be predicted from a single time point alone, we consider the maximum tolerable image degradation and distribute this value over the longest likely duration of the motion periods.
According to motion behaviors reported from epileptic patients, ${ }^{17}$ a maximum of 49 jerks may be expected over the duration of the acquisition of 1 image volume in the present study (7 min $21 \mathrm{~s}$ ). This corresponds to a maximum of $147 \mathrm{~s}$ of movement during our acquisition ( $3 \mathrm{~s}$ per jerk), equivalent to the acquisition of $147 \mathrm{~s} / \mathrm{TR}=6000 \mathrm{k}$-space readout lines. Given the maximum aggregate value of $\overline{\Delta \mathrm{Mew}}$ observed in our experiments $(\sim 12 \mathrm{~mm} / \mathrm{s})$ (see Figure 2$)$, the largest (instantaneous) value of $\Delta \mathrm{Mew}_{\mathrm{i}}$ may be $12 / 6000=2 \mathrm{e}^{-3} \mathrm{~mm} /$ s. Motion experiments were therefore conducted with suspension of data acquisition when $\Delta \mathrm{Mew}_{\mathrm{i}}$ was exceeding threshold values $\Delta \mathrm{Mew}_{\mathrm{th}}=1.75 \mathrm{e}^{-4}, 3.5 \mathrm{e}^{-4}, 7 \mathrm{e}^{-4}, 1.4 \mathrm{e}^{-3}$, and $2.8 \mathrm{e}^{-3} \mathrm{~mm} / \mathrm{s}$, to cover all possible scenarios of patients motion behavior.

Note that suspending data acquisition based on the value of $\Delta \mathrm{Mew}_{\mathrm{i}}$ is equivalent to suspending the acquisition based on the head speed according to: $S_{i}>\frac{\Delta M e w_{t h}+M e w_{\text {compliant }, i}}{W_{i}}$ (Figure $1 \mathrm{E})$. With $\Delta \mathrm{Mew}_{\mathrm{th}}=0$, the suspension is most stringent: any head speed beyond those of the compliant population leads to suspension of data acquisition, regardless of the location in $\mathrm{k}$-space. With $\Delta \mathrm{Mew}_{\mathrm{th}}>0$, the threshold value of the head speed, $\mathrm{S}_{\mathrm{th}}$, depends on the amplitude of the signal acquired at the time of the motion (see Figure $1 \mathrm{E}$ ): $S_{\mathrm{th}}$ is high/low when the amplitude of the acquired signal is low/ high. Note that to avoid excessive additional scan times in case of continuous participant motion, the total duration of the suspension period was not allowed to exceed the nominal duration of the scans. Upon reaching this limit, the scanner was required to resume scanning without further suspension, leading to a doubling of the acquisition time. This situation was never encountered in our experiments.

\section{3 | Marker of image degradation}

Maps of the MRI parameter $\mathrm{R}_{2}^{*}$ were computed from the PDw data acquired in the motion and no-motion conditions (see data acquisition and motion experiments below). The $\mathrm{R}_{2}^{*}$ maps were obtained by voxel-wise linear fitting of the log of the signal amplitudes across echoes, as described in Refs.18,19. Because head motion leads to deviations from the exponential decays, ${ }^{20,21}$ maps of the SD of the residuals $\varepsilon$ (standard error of the $\mathrm{R}_{2}^{*}$ estimates) were calculated as measures of the goodness of fit of the monoexponential model. When low levels of motion artefact are present in the MRI data, $\mathrm{R}_{2}^{*}$ values are known correlates of iron and myelin concentration in brain tissue. ${ }^{22-24} \mathrm{R}_{2}^{*}$ maps are known to be particularly sensitive to motion. ${ }^{20}$ Head motion increases the spatial variability of $\mathrm{R}_{2}^{*}$ maps, and region-specific coefficients of variation of $\mathrm{R}_{2}^{*}$ have been shown to characterize image degradation due to head motion. ${ }^{10}$ Building on this idea, we used estimates of the $\mathrm{SDR}_{2}^{*}$ values in white matter as markers of motion artefact levels in the acquired data. The white matter masks were calculated by segmenting the magnetization 
A

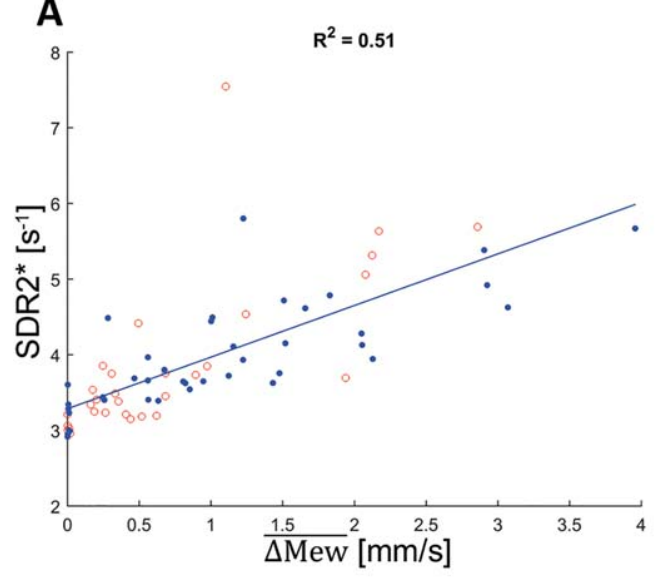

B

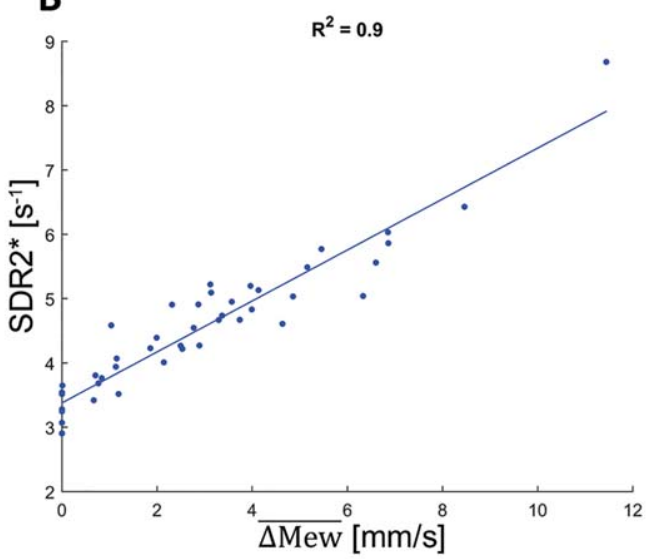

C

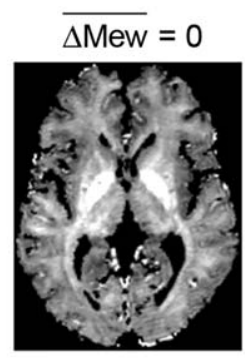

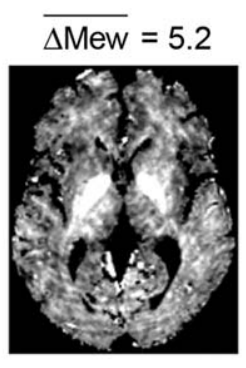

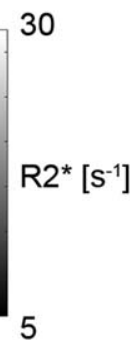

F I G URE 2 Relationship between image degradation ( $\mathrm{SDR}_{2}^{*}$ ) and the aggregate measure of motion $\overline{\Delta \mathrm{Mew}}$ (no suspension of data acquisition). (A) Results of experiments 1 (red: empty circles) and 2 (blue: solid circles) acquired with the 64-channel head coil. (B) Results of experiment 2 acquired with the 20-channel head coil. (C) Example $\mathrm{R}_{2}^{*}$ maps acquired under motion experiments leading to different $\overline{\Delta \mathrm{Mew}}$ values.

$\overline{\Delta \mathrm{Mew}}$, aggregate measure of motion; $\mathrm{SDR}_{2}, \mathrm{SD}$ of the $\mathrm{R}_{2}^{*}$ values

transfer maps using SPM's unified segmentation ${ }^{25}$ and including voxels with a minimal white matter probability of 0.95 . The white matter masks were eroded by 2 voxels to reduce the contribution of regions of inhomogeneous $B_{0}$ field to the $\mathrm{SDR}_{2}^{*}$ estimates. This white matter mask was also used to calculate the mean $\varepsilon$ estimates in white matter.

\section{4 | Data acquisition}

Data were acquired on 5 participants on a 3T Siemens Prisma MRI scanner (Erlangen, Germany) using a 64channel and a 20-channel head-neck coil and a custom-made 3D FLASH acquisition. ${ }^{18,26}$ The k-space trajectory of the FLASH sequence was Cartesian-centered (i.e., the center of $\mathrm{k}$-space was acquired halfway through the acquisition). The image resolution was $1.5 \mathrm{~mm}$ isotropically, leading to a voxel size of $1.5^{3} \mathrm{~mm}^{3}$. The matrix size was $160 \times 150 \times 120$, and the acquisition time was $7 \min 21 \mathrm{~s}$ per image volume. The TR was $24.5 \mathrm{~ms}$. Eight echo images were acquired with a bipolar readout (readout bandwidth: $466 \mathrm{~Hz} /$ pixel) following each (nonselective) RF excitation, with TEs ranging from $2.34 \mathrm{~ms}$ to $18.72 \mathrm{~ms}$ in steps of $2.34 \mathrm{~ms}$.

Prior to the motion experiments (see below), subjects were requested to remain still. During this period of no motion, data were acquired with proton density (PDw), $\mathrm{T}_{1}$ $\left(\mathrm{T}_{1} \mathrm{w}\right)$, and magnetization transfer-weighted contrast ( $\mathrm{RF}$ excitation flip angles: $6^{\circ}, 21^{\circ}, 6^{\circ}$, respectively). ${ }^{18}$ Magnetization transfer-weighting was achieved using an off-resonance Gaussian-shaped RF pulse applied prior to RF excitation, typical amplitude: $\sim 150$ to $200 \mathrm{~V}$, duration: $4 \mathrm{~ms}$, nominal flip angle: $220^{\circ}$, frequency offset from water resonance: $2 \mathrm{k}$ $\mathrm{Hz}^{18} \mathrm{~B}_{1}$ mapping data ${ }^{27,28}$ was used together with the FLASH data to compute quantitative magnetization transfer maps offline according to Ref. 29. Only PDw data was acquired for each condition of the motion experiments.

Data acquisition was repeated across multiple scanning sessions for each motion experiment, with and without suspension of data acquisition and with different head coils. The subjects were taken out of the MRI scanner between sessions, and scanner calibration (e.g., shimming) was only conducted at the start of each session.

\section{5 | Prospective motion correction}

$\mathrm{PMC}^{6}$ was used for all acquisitions conducted in this study. Tracking was performed by an optical camera and a Moiré Phase Tracking marker (Metria Innovation Inc., WI, Wauwatosa). The camera was mounted inside the MRI scanner bore (see Figure $1 b$ in Ref. 9). To ensure that the motion of the marker was an accurate reflection of head motion, the marker was attached to a mouthpiece fitted to the dental print of each participant by a dental technician. The marker position 
TABLE 1 Summary table of the motion experiments, receive head coils, and $\Delta \mathrm{Mew}_{\text {th }}$ values used in the present study

\begin{tabular}{|c|c|c|}
\hline & $\begin{array}{l}\text { 64-Channel Head } \\
\text { Coil }\end{array}$ & $\begin{array}{l}\text { 20-Channel Head } \\
\text { Coil }\end{array}$ \\
\hline $\begin{array}{l}\text { Experiment } 1: 44 \mathrm{~s} \\
\text { motion periods: } \\
\text { variable onset } \\
\text { times }\end{array}$ & $\begin{array}{c}\text { Data suspension OFF } \\
\Delta \mathrm{Mew}_{\text {th }}=0,7 \mathrm{e}^{-4}\end{array}$ & \\
\hline $\begin{array}{l}\text { Experiment } 2: 3 \mathrm{~s} \\
\text { motion periods } \\
\text { (“jerks") Variable } \\
\text { number of jerks }\end{array}$ & $\begin{array}{l}\text { Data suspension OFF } \\
\qquad \mathrm{Mew}_{\text {th }}=1.75 \mathrm{e}^{-4} \\
3.5 \mathrm{e}^{-4}, 7 \mathrm{e}^{-4}, 1.4 \mathrm{e}^{-3}, \\
\text { and } 2.8 \mathrm{e}^{-3}\end{array}$ & $\begin{array}{l}\text { Data suspension OFF } \\
\Delta \mathrm{Mew}_{\text {th }}=1,75 \mathrm{e}^{-4}, \\
3.5 \mathrm{e}^{-4}, 7 \mathrm{e}^{-4}, \\
1.4 \mathrm{e}^{-3}, \text { and } 2.8 \mathrm{e}^{-3}\end{array}$ \\
\hline
\end{tabular}

detected by the camera (tracking accuracy $\sim 0.01 \mathrm{~mm}$ in translation and $\sim 0.01^{\circ}$ in rotation ${ }^{9}$ ) was sent in real time to the MRI host computer to adjust the MRI acquisition. ${ }^{9}$ Before each experiment, the participants were asked to move in all directions to ensure optimal coverage of the marker position by the camera. As a precautionary measure, the MRI acquisition sequence was programmed to suspend data acquisition when the marker position was not available (indicating that the marker was out of the FOV). The largest translations and rotations observed during the experiments with respect to the initial pose were $x=9.9 \mathrm{~mm}, y=6.8 \mathrm{~mm}$, $z=7.3 \mathrm{~mm}, R x=9.2^{\circ}, R y=8.1^{\circ}$ and $R z=9.1^{\circ}$.

Adjustment of the MRI scanner components was implemented according to the latest head position information available from the camera prior to each RF pulse (frequency $=1$ / TR). Note that RF excitation was maintained during periods of suspension of data acquisition to preserve the steady-state equilibrium of the magnetization. Data acquisition was suspended when the marker was out of the FOV of the camera to ensure optimal motion correction of all data points.

\section{6 | Motion experiment}

\subsection{1 | Experiment 1}

The volunteers were instructed to move during periods of 44 $\mathrm{s}$, that is, $10 \%$ of the image acquisition time. The motion experiments were conducted using 5 conditions, which covered the first, second, third, fourth, and fifth $10 \%$ of $\mathrm{k}$-space. As a result, condition 5 involved motion during the acquisition of the center of k-space, leading to maximum image degradation. This experiment was performed using a 64channel head coil (see Table 1).

\subsection{2 | Experiment 2}

The volunteers were instructed to move during periods of 3 seconds (jerks) to replicate motion behaviors reported from epileptic patients. ${ }^{17}$ Data acquisition was repeated for 4 conditions, with 10, 20, 30, and 40 jerks per acquisition. The onset times of the jerks were randomized for each acquisition. This experiment was performed using a 64- and 20channel head coil (see Table 1).

For both motion experiments 1 and 2, the order of the motion conditions was randomized. No instructions were given to the participants on the type of motion that they should carry out. Both experiments were first conducted with no interruption of data acquisition (but with PMC) to study the
A
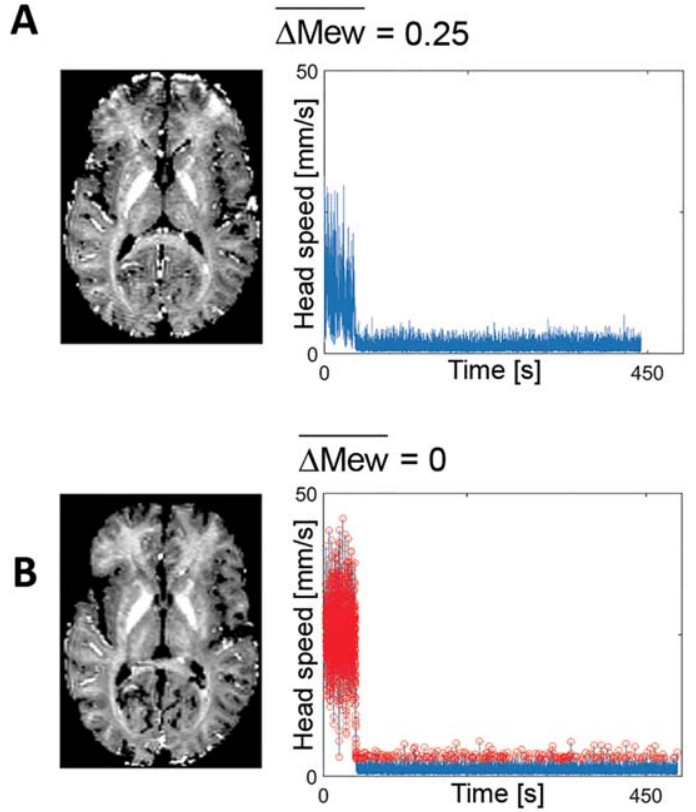

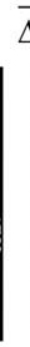

$\overline{\Delta \mathrm{Mew}}=2.12$
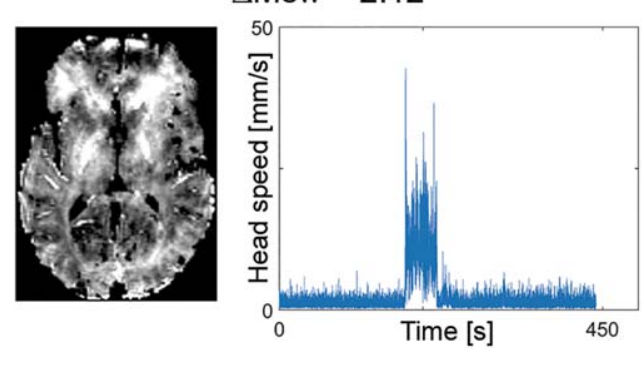

$\overline{\Delta \mathrm{Mew}}=0$

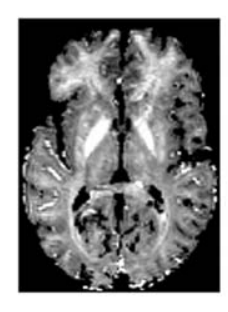

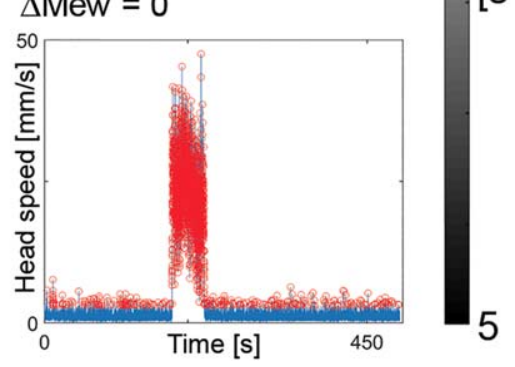

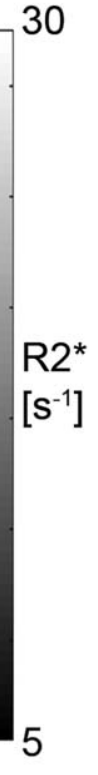

F I G URE 3 Example of $\mathrm{R}_{2}^{*}$ maps acquired without (A) and with (B) suspension of data acquisition during periods of head motion (experiment 1). In (B), the threshold value used for suspension of the acquisition was $\Delta \mathrm{Mew}_{\mathrm{th}}=0$ and the points of suspended acquisition are highlighted in red (11\% and $12 \%$ of the total number of points). The aggregate measures of motion impact, calculated from the motion trajectories, are indicated on top of each $\mathrm{R}_{2}^{*}$ map 
TABLE 2 Additional scan time (in percent) due to the suspension of data acquisition and corresponding SDR 2 values for the motion conditions of experiment 1

\begin{tabular}{|c|c|c|c|c|c|c|}
\hline & & $\begin{array}{l}\text { Condition } 1 \\
(0 \%-10 \% \\
\text { of k-space) }\end{array}$ & $\begin{array}{l}\text { Condition } 2 \\
(10 \%-20 \% \\
\text { of k-space) }\end{array}$ & $\begin{array}{l}\text { Condition } 3 \\
(20 \%-30 \% \\
\text { of k-space) }\end{array}$ & $\begin{array}{l}\text { Condition } 4 \\
(30 \%-40 \% \\
\text { of k-space) }\end{array}$ & $\begin{array}{l}\text { Condition } 5 \\
(40 \%-50 \% \\
\text { of k-space) }\end{array}$ \\
\hline \multirow[t]{2}{*}{$\Delta \mathrm{Mew}_{\mathrm{th}}=0$} & Additional time [\%] & 10.4 & 11.2 & 11.4 & 11.1 & 11.1 \\
\hline & $\mathrm{SDR}_{2}^{*}\left[\mathrm{~s}^{-1}\right]$ & 3.01 & 3.09 & 2.99 & 3.06 & 3.22 \\
\hline \multirow[t]{2}{*}{$\Delta \mathrm{Mew}_{\text {th }}=7 \mathrm{e}^{-4}$} & Additional time [\%] & 1.6 & 3.9 & 4.3 & 6.8 & 8.4 \\
\hline & $\mathrm{SDR}_{2}^{*}\left[\mathrm{~s}^{-1}\right]$ & 3.16 & 3.27 & 3.32 & 3.34 & 3.48 \\
\hline
\end{tabular}

$\Delta \mathrm{Mew}_{\text {th }}$, encoding-weighted integrated motion metric; $\mathrm{SDR}_{2}^{*}, \mathrm{SD}$ of $\mathrm{R}_{2}^{*}$ values.

relationship between motion history and degradation of the $\mathrm{R}_{2}^{*}$ maps $\left(\mathrm{SDR}_{2}^{*}\right)$, despite conventional PMC. From this relationship, a predictor of the impact of motion on image quality was identified. Both experiments were then repeated with interruption of data acquisition when different threshold values of this predictor were exceeded. Note that 1 subject did not take part in the experiments with acquisition suspension.

\section{3 | RESULTS}

Without suspension of data acquisition, the degradation of the $\mathrm{R}_{2}^{*}$ maps with head motion follows the same linear dependence on $\overline{\Delta \mathrm{Mew}}$ for all subjects and under both block and jerk-like motion conditions (Figures 2A, B). This was observed for both head coils, although less robustly for the 64-channel coil $\left(\mathrm{R}^{2}=0.51\right.$, slope $\left.=0.68 \mathrm{~mm}^{-1}\right)$ than for the 20-channel coil $\left(\mathrm{R}^{2}=0.9\right.$, slope $\left.=0.39 \mathrm{~mm}^{-1}\right)$. Figure $2 \mathrm{C}$ shows example $R_{2}^{*}$ maps for different values of $\overline{\Delta \mathrm{Mew}}$. The degradation of the $R_{2}^{*}$ maps increases when motion takes place near the center of $\mathrm{k}$-space (experiment 1 ) or with an increasing number of jerks (experiment 2). The good agreement of the relationship between $\overline{\Delta \mathrm{Mew}}$ and $\mathrm{SDR}_{2}^{*}$ for both motion experiments establishes $\overline{\Delta \mathrm{Mew}}$ as a summary measure of motion impact, aggregated over the whole acquisition, regardless of the motion trajectories.
A

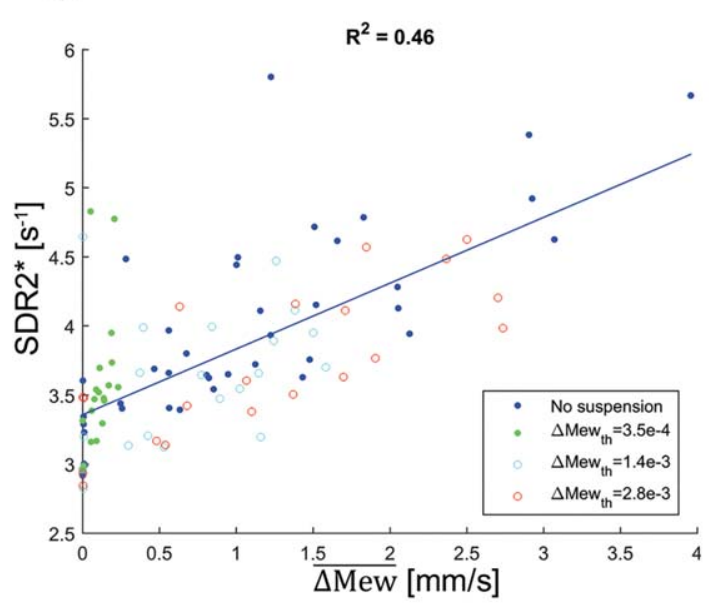

B

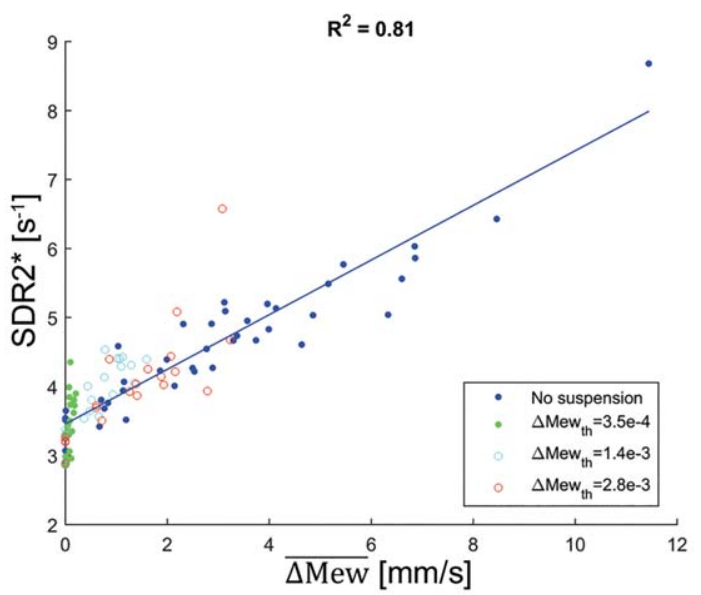

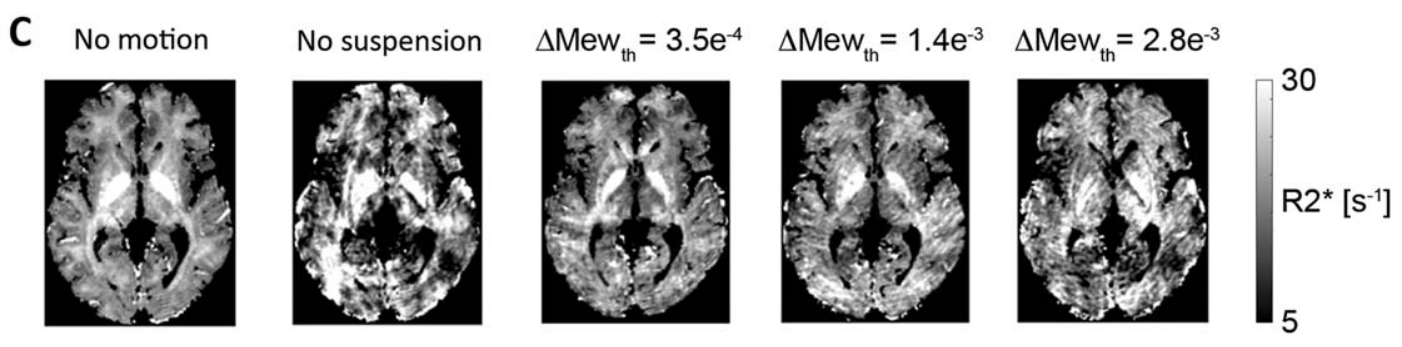

FIG URE 4 Relationship between image degradation $\left(\mathrm{SDR}_{2}^{*}\right)$ and the aggregate measure of motion $\overline{\Delta \mathrm{Mew}}$ for experiment 2 with and without acquisition suspension. Data was acquired with the 64-channel (A) and 20-channel (B) head coils. (C) $R_{2}^{*}$ maps corresponding to the highest $S D R_{2}^{*}$ values obtained for each threshold value of the acquisition suspension with the 20-channel head coil 
A
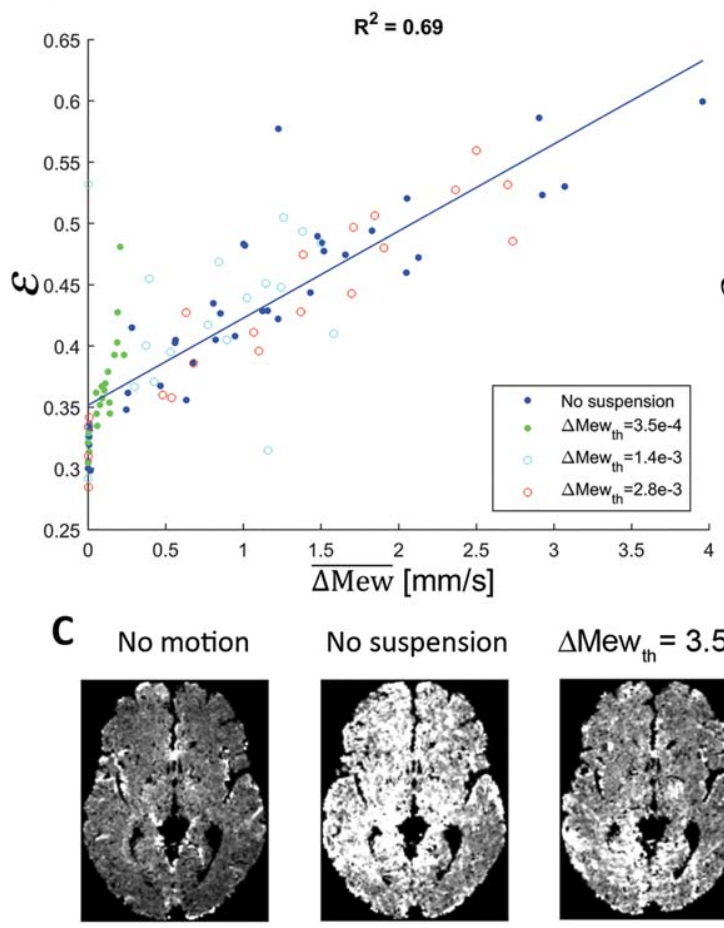

No suspension

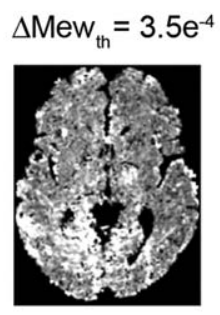

\section{B}

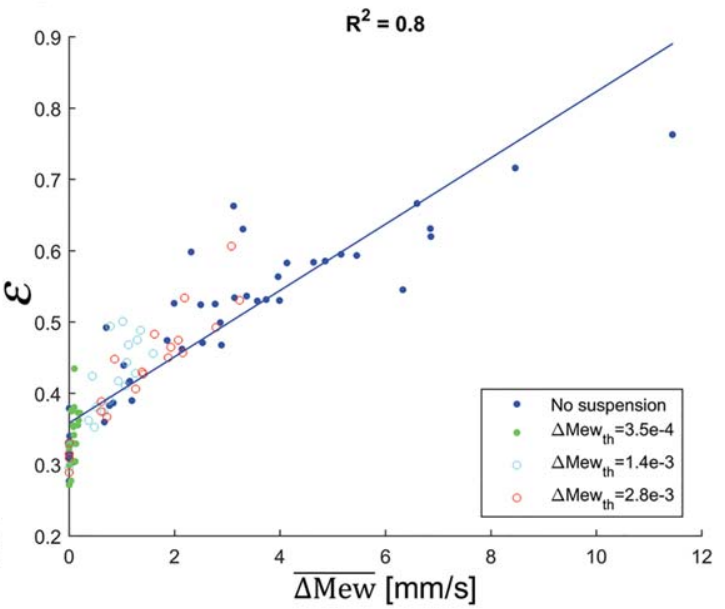

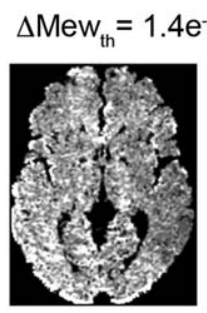

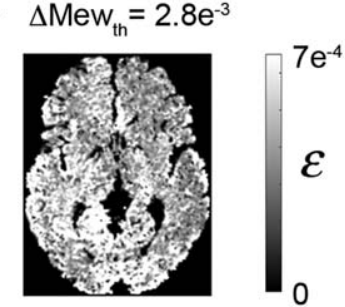

FIG URE 5 Relationship between mean residuals of the $\mathrm{R}_{2}^{*}$ fit (c) and the aggregate measure of motion $\overline{\Delta M e w}$ for experiment 2 with and without acquisition suspension. Data was acquired with the 64-channel (A) and 20-channel (B) head coils. (C) Residuals maps corresponding to the highest SDR 2 values obtained for each threshold value of the acquisition suspension with the 20-channel head coil

Figure 3 shows example $\mathrm{R}_{2}^{*}$ maps acquired with PMC but without (Figure $3 \mathrm{~A})$ and with $\left(\Delta \mathrm{Mew}_{\mathrm{th}}=0 \mathrm{~mm} / \mathrm{s}\right)$ (Figure 3B) suspension of data acquisition (motion experiment 1 , onset time of the motion period: $0 \mathrm{~s}$ and $176 \mathrm{~s}$ ). An example $\mathrm{R}_{2}^{*}$ map acquired without motion period is available in Figure 2 for reference. In Figure 3B, the time points corresponding to suspension of data acquisition are highlighted in red and accounted for $11 \%$ and $12 \%$ of the total number of points. The suspension of data acquisition leads to the largest improvements in data quality when motion takes place during the acquisition of the center of k-space. The quality of the $R_{2}^{*}$ maps under the motion conditions remains close to that of the $\mathrm{R}_{2}^{*}$ maps acquired without motion. Table 2 shows the increase in scan time due to the suspension of data acquisition and the corresponding values of $\mathrm{SDR}_{2}^{*}$ (experiment 1 ). With $\Delta \mathrm{Mew}_{\mathrm{th}}=0 \mathrm{~mm} / \mathrm{s}$, optimal image quality is preserved for all motion conditions at the cost of a large increase in acquisition time (45 s-50 s, the duration of the motion period in experiment 1). When $\Delta \mathrm{Mew}_{\mathrm{th}}=7 \mathrm{e}^{-4} \mathrm{~mm} / \mathrm{s}$, increasing image degradation is observed as motion takes place near the center of $\mathrm{k}$-space. The additional scan duration is short/long when motion takes place at the periphery/center of $\mathrm{k}$-space (conditions 1 of 5).

Figures $4 \mathrm{~A}$ and $4 \mathrm{~B}$ show the effect of suspending data acquisition for $\Delta \mathrm{Mew}_{\mathrm{th}}=3.5 \mathrm{e}^{-4}, 1.4 \mathrm{e}^{-3}$, and $2.8 \mathrm{e}^{-3} \mathrm{~mm} / \mathrm{s}$. For a given motion condition, the maximum $\overline{\Delta \mathrm{Mew}}$ and $\mathrm{SDR}_{2}^{*}$ values decrease when $\Delta \mathrm{Mew}_{\mathrm{th}}$ is reduced, illustrating a gain in image quality. As for Figure 2, the robustness of the linear dependence of $\mathrm{SDR}_{2}^{*}$ on $\overline{\Delta \mathrm{MeW}}$ is less pronounced for the 64-channel coil $\left(R^{2}=0.46\right)$ than for the 20-channel coil $\left(R^{2}=0.81\right)$. Figure $4 C$ shows the $R_{2}^{*}$ maps with the highest $\mathrm{SDR}_{2}^{*}$ for each threshold value (20-channel coil and experiment 2). The improvement in image quality when $\Delta \mathrm{Mew}_{\mathrm{th}}$ is reduced is clearly apparent. For $\Delta \mathrm{Mew}_{\mathrm{th}}=3.5 \mathrm{e}^{-4} \mathrm{~mm} / \mathrm{s}$, the quality of the $\mathrm{R}_{2}^{*}$ maps $\left(\mathrm{SDR}_{2}^{*}=4.35 \mathrm{~s}^{-1} ; \overline{\Delta \mathrm{Mew}}=1 \mathrm{e}^{-1} \mathrm{~mm} /\right.$ $\mathrm{s})$ is close to that obtained under the no motion condition $\left(\mathrm{SDR}_{2}^{*}=3.08 \mathrm{~s}^{-1} ; \overline{\Delta \mathrm{Mew}}=1 \mathrm{e}^{-3} \mathrm{~mm} / \mathrm{s}\right)$.

Figures $5 \mathrm{~A}$ and $5 \mathrm{~B}$ show the relationship between the residuals $\varepsilon$ and $\overline{\Delta \mathrm{Mew}}$ for $\Delta \mathrm{Mew}_{\mathrm{th}}=3.5 \mathrm{e}^{-4}, 1.4 \mathrm{e}^{-3}$, and $2.8 \mathrm{e}^{-3}$ $\mathrm{mm} / \mathrm{s}$. Figure $5 \mathrm{C}$ shows the maps of $\varepsilon$ corresponding to the highest $\mathrm{SDR}_{2}^{*}$ values of $\Delta \mathrm{Mew}_{\text {th }}$. Without suspension of the acquisition, $\varepsilon$ follows a similar linear dependence on $\overline{\Delta \mathrm{Mew}}$ as $\mathrm{SDR}_{2}^{*}$ (Figure 4). For low values of $\Delta \mathrm{Mew}_{\mathrm{th}}$, the values of $\varepsilon$ tend to remain near the values of the no-motion condition. The range of $\varepsilon$ values increases with increasing $\Delta \mathrm{Mew}_{\text {th }}$ but remains smaller than those obtained without acquisition suspension.

\section{4 | DISCUSSION}

In this study, we improve the quality of MRI data acquired with prospective motion correction by suspending data acquisition during periods of detrimental motion. We present 
an aggregate measure of motion history during the acquisition that exhibits a high correlation with the degradation of the resulting image (Figure 2). From this predictor of motion impact, a threshold parameter is defined to suspend image acquisition during motion periods (Figure 3 ). This threshold guarantees a minimum level of data quality in the final images, given the motion characteristics of patient populations (Figure 4). This threshold also governs the tradeoff between image quality and the prolongation of scan time due to the suspension of the acquisition (Table 2) and can be set by the user depending on the desired level of image quality and patient tolerance for prolonged scan durations.

The relationship between the aggregate measure of motion and image degradation (Figure 2) was examined for 2 types of head movements: continuous motion periods of 44 $\mathrm{s}$ with predefined onset times (experiment 1) and short motion periods of $3 \mathrm{~s}$ (jerks) at random times during the scans (experiment 2). The comparability of the participant's motion behavior between motion conditions was not a prerequisite for this study: no instructions were given to the participants regarding their motion behaviors, and in experiment 2 , the onset times of the jerks were randomized across repetitions. Despite this variability, the relationship between image degradation and the motion impact remained remarkably consistent, suggesting that the predictor of motion impact may hold for a large range of motion behaviors (this relationship was preserved when the TR duration of the acquisition was extended to $50 \mathrm{~ms}$; data not shown). However, the correlation between image degradation and motion impact was reduced with the 64-channel coil. The numerical simulation results presented in Supporting Information Figure S2 suggest that head motion in the sharp sensitivity profile of this coil might be the origin of the effect, which is in line with recent literature. $^{30,31}$ The delay between tracking of the marker position and acquisition update on the measures of $\overline{\Delta \mathrm{Mew}}\left(\sim\right.$ twice our TR value ${ }^{6}$ ) was estimated to lead to an average (SD) change in $\overline{\Delta \mathrm{Mew}}$ of $0.017(0.030) \mathrm{mm} / \mathrm{s}$, small compared to the range of $\overline{\Delta \mathrm{Mew}}$ values encountered due to motion (see Supporting Information Figure S3).

Aside from head speed, the predictor of motion impact accounts for the time of occurrence of the motion in k-space. As a result, periods of strong motion may be tolerated at innocuous times of the image encoding order to minimize the increase in acquisition time at a minimal cost in terms of image quality. The prolongation of scan time due to the suspension of data acquisition was estimated from experiment 1 , for which the motion behavior may be assumed reproducible across multiple repetitions of the same condition. The results are presented in Table 2. Suspension of data acquisition based on head speed only $\left(\Delta \mathrm{M}_{\text {ewth }}=0 \mathrm{~mm} / \mathrm{s}\right)$ led to systematic prolongations of scan time by the duration of the motion periods. With $\Delta \mathrm{M}_{\mathrm{ewth}}=7 \mathrm{e}^{-4} \mathrm{~mm} / \mathrm{s}$, the prolongation of scan time varied by a factor 5 , depending on the time of occurrence of the motion.

Although the presented framework for suspension of data acquisition during periods of detrimental head motion was designed for FLASH, it might be extended to other types of image acquisitions. However, suspension of data acquisition might in general not be recommended for functional MRI, for which the synchronicity of image acquisition and functional stimulation is paramount. The proposed framework is particularly well suited for acquisitions, for which each head motion information used for prospective correction can be associated with data of similar amplitude. However, specific adjustments might be required for each type of acquisition (e.g., FLAIR, MPRAGE). In the context of EPI acquisitions, this would require new motion information at the frequency of the EPI readout sampling $(\sim 1 \mathrm{kHz})$ - out of range of our system-and would also require careful implementation to prevent the occurrence of image ghosting. However, an implementation based on head speed only, prior to RF excitation, is likely to bring significant improvements in data quality (e.g., diffusion MRI) in noncompliant populations. Note that keeping the time interval between consecutive RF excitations constant for a given slice is critical for 2D acquisitions. A direct implementation of the proposed framework, for which RF excitation is maintained during periods of acquisition suspension, might violate this principle depending on the acquisition scheme used. An alternative strategy, as discussed below, is to complete data acquisition despite the occurrence of motion and to reacquire the data points affected by motion post-hoc while preserving the slice ordering of the acquisition.

In the presented framework, the weights used to calculate the predictor of motion impact $\overline{\Delta \mathrm{Mew}}$ were taken as an average across an independent population. In Supporting Information Figure S4, these $\overline{\Delta \mathrm{Mew}}$ estimates are compared to those obtained from the individual weights of the participants to the motion experiments. Although individual weights cannot not be used in practice to suspend the acquisition, these results provide a measure of the accuracy of the $\overline{\Delta \mathrm{Mew}}$ estimates calculated with the proposed framework. The mean (SD) difference between average and individual weights was $-15.94(23.04) \%$. The average (SD) deviation between the $\overline{\Delta \mathrm{Mew}}$ values obtained from the average and individual weights was $0.031(0.062) \mathrm{mm} / \mathrm{s}$. The largest deviations were obtained for high values of $\Delta \mathrm{Mew}_{\mathrm{th}}$, which preferentially targets data points at the k-space center (see Supporting Information Figure S4).

The use of weights averaged across a reference population requires acquisition of data on a compliant cohort prior to implementation of the proposed framework. Although this task needs to be completed only once, an alternative approach based on the relationship between the amplitude of the weights and their distance to the origin of k-space might 
be preferable. Based on the observed relationship (see Supporting Information Figure S5A), alternative weights were computed and used in offline analysis to calculate new estimates of $\overline{\Delta \mathrm{Mew}}$ from the motion trajectories recorded during the experiments. The results are presented in Supporting Information Figure S5B-D. The average (SD) difference between the $\overline{\Delta \mathrm{Mew}}$ values calculated from the individual weights (our gold standard for accuracy assessment) and these alternative weights was $0.048(0.087) \mathrm{mm} / \mathrm{s}$. This is $\sim 50 \%$ larger than the accuracy estimates reported above for the proposed framework but remains small compared to the range of $\overline{\Delta \mathrm{Mew}}$ values encountered due to motion.

This study illustrates the improvements in data quality when low spatial frequencies are preferentially targeted to trigger the suspension of data acquisition (Figure 4). These results open the way for follow-up studies comparing the real-time data suspension presented here with other strategies to minimize motion impact. On the model of previous works, ${ }^{32-34} 1$ candidate approach is to adjust the $\mathrm{k}$-space trajectory of the acquisition in real time depending on the motion behavior. With this approach, regions of $\mathrm{k}$-space with low signal amplitude (high k-space values) would be acquired during periods of head motion, with little effect on image quality. This approach would alleviate the need for suspension of data acquisition, keeping the acquisition time minimal for all motion behaviors. Another alternative, using the theoretical framework presented here, would be to complete the acquisition of the whole image without suspension and re-acquire post-hoc the k-space lines most affected by motion until a target $\overline{\Delta \mathrm{Mew}}$ is reached. This strategy has been implemented in previous studies by identifying $\mathrm{k}$-space lines corrupted by motion based on motion score ${ }^{12,13}$ or magnitude/phase data. ${ }^{15,16}$ This approach would circumvent the need for assumptions on the expected amount of head motion to determine threshold values from aggregate measures of motion impact (see real-time suspension of data acquisition during periods of motion section above), and would adjust naturally to the different motion behaviors encountered. Note that this strategy implies that the acquisition of neighboring data points might be separated by long time intervals, which could degrade the phase consistency of neighboring k-space lines and exacerbate image degradation (e.g., ghosting) when dynamic effects are present during the acquisition. ${ }^{32,33}$

Here, we use the $\mathrm{SD}$ of $\mathrm{R}_{2}^{*}$ maps in white matter $\left(\mathrm{SDR}_{2}^{*}\right)$ as a marker of image quality. We chose this parameter because it can be computed with ease, because of its sensitivity to motion-related image degradation, ${ }^{10,20}$ and because quantitative maps of MRI parameters do not suffer from the sources of image bias that commonly affect anatomical MRI data. ${ }^{18}$ Other markers of motion artefacts might be equally suitable. ${ }^{35} \mathrm{R}_{2}^{*}$ maps are sensitive to the orientation of the head relative to the main magnetic field ${ }^{6}$ and to the inhomogeneities of this field near the air-tissue interface. ${ }^{37}$ The resulting variability in the $\mathrm{SDR}_{2}^{*}$ values can be estimated to be up to $1 \mathrm{~s}^{-1}$ from the data acquired across multiple scanning sessions in the absence of head motion. This is about 5 times smaller than changes in $\mathrm{SDR}_{2}^{*}$ due to head motion, which shows a remarkable level of correlation with $\overline{\Delta \mathrm{Mew}}$ nonetheless. Note that the contribution of voxels near the air-tissue interface was minimized by eroding the white matter masks by 2 voxels prior to the calculation of the $\mathrm{SDR}_{2}^{*}$ estimates.

On top of motivating the use of $\overline{\Delta \mathrm{Mew}}$ for suspension of data acquisition in real time, the linear increase in $\mathrm{SDR}_{2}^{*}$ with $\overline{\Delta \mathrm{Mew}}$ also suggests that $\mathrm{SDR}_{2}^{*}$ might be used as an objective measure of data quality, potentially more robust than visual assessment. However, it should be emphasized that $\mathrm{SDR}_{2}^{*}$ depends on the level of noise in the acquired data (image resolution) and on the robustness of the $R_{2}^{*}$ fitting (number of echoes). It should also be noted that because $\mathrm{R}_{2}^{*}$ is a biomarker of the microstructural properties of brain tissue, ${ }^{22-24} \mathrm{SDR}_{2}^{*}$ values might be affected by microscopic changes in the healthy and diseased brain.

\section{5 | CONCLUSION}

We present a framework that allows the suspension of MRI data acquisition during periods of head motion. In this framework, head motion information is provided by a prospective motion correction system during the scans and is processed in real time to compute a predictor of the impact of motion on the MR images. Data acquisition is suspended when excessive motion degradation is predicted. The predictor of motion impact accounts for both head speed and the amplitude of the k-space signal acquired at the time of the motion, which allows for a more efficient reacquisition strategy than that solely based on head speed. The framework was tested for 2 different kinds of motion behavior. Suspending data acquisition during impactful periods of head motion led to significant improvements in the quality of the MRI images. The tradeoff between image degradation due to motion and prolongation of scan time due to the suspension of data acquisition may be tuned depending on the desired MRI image quality and subject tolerability.

\section{ACKNOWLEDGMENTS}

This work was carried out on the MRI platform of the Département des neurosciences cliniques-Centre hospitalier universitaire vaudois, which is generously supported by the Roger De Spoelberch and Partridge Foundations. A.L. is supported by the Roger De Spoelberch Foundation. B.D. is supported by the Swiss National Science Foundation (NCCR Synapsy, project grant Nr 32003B_159780), Foundation Parkinson Switzerland, and Foundation Synapsis. The research leading to these results has received funding 
from the European Union's Horizon 2020 research and innovation program under grant agreement No. 720270 (HBP SGA1). The authors would like to thank Dr. David Carmichael (Institute of Child Health, University College London, UK) for helpful discussions on the movement characteristics of epileptic patients.

\section{ORCID}

Rémi Castella (1D http://orcid.org/0000-0002-4256-3552 Antoine Lutti (1) http://orcid.org/0000-0003-3281-5477

\section{REFERENCES}

[1] Andre JB, Bresnahan BW, Mossa-Basha M, et al. Toward quantifying the prevalence, severity, and cost associated with patient motion during clinical MR examinations. J Am Coll Radiol. 2015;12:689-695.

[2] Reuter M, Tisdall MD, Qureshi A, Buckner RL, van der Kouwe AJW, Fischl B. Head motion during MRI acquisition reduces gray matter volume and thickness estimates. Neuroimage. 2015; 107:107-115.

[3] Yendiki A, Koldewyn K, Kakunoori S, Kanwisher N, Fischl B. Spurious group differences due to head motion in a diffusion MRI study. Neuroimage. 2014;88:79-90.

[4] Todd N, Josephs O, Callaghan MF, Lutti A, Weiskopf N. Prospective motion correction of 3D echo-planar imaging data for functional MRI using optical tracking. Neuroimage. 2015;113:112.

[5] Godenschweger F, Kägebein U, Stucht D, et al. Motion correction in MRI of the brain. Phys Med Biol. 2016;61:R32-R56.

[6] Zaitsev M, Dold C, Sakas G, Hennig J, Speck O. Magnetic resonance imaging of freely moving objects: prospective real-time motion correction using an external optical motion tracking system. Neuroimage. 2006;31:1038-1050.

[7] Maclaren J, Herbst M, Speck O, Zaitsev M. Prospective motion correction in brain imaging: a review. Magn Reson Med. 2013; 69:621-636.

[8] Yancey SE, Rotenberg DJ, Tam F, et al. Spin-history artifact during functional MRI: Potential for adaptive correction. Med Phys. 2011;38:4634.

[9] Maclaren J, Armstrong BSR, Barrows RT, et al. Measurement and correction of microscopic head motion during magnetic resonance imaging of the brain. PLoS One. 2012;7:e48088.

[10] Callaghan MF, Josephs O, Herbst M, Zaitsev M, Todd N, Weiskopf N. An evaluation of prospective motion correction (PMC) for high resolution quantitative MRI. Front Neurosci. 2015;9:97.

[11] Aksoy M, Forman C, Straka M, Çukur T, Hornegger J, Bammer R. Hybrid prospective and retrospective head motion correction to mitigate cross-calibration errors. Magn Reson Med. 2012;67:1237-1251.

[12] Frost R, Hess AT, Okell TW, et al. Prospective motion correction and selective reacquisition using volumetric navigators for vessel-encoded arterial spin labeling dynamic angiography. Magn Reson Med 2016;76:1420-1430.

[13] Tisdall MD, Hess AT, Reuter M, Meintjes EM, Fischl B, Van Der Kouwe AJW. Volumetric navigators for prospective motion correction and selective reacquisition in neuroanatomical MRI. Magn Reson Med. 2012;68:389-399.

[14] Bogner W, Gagoski B, Hess AT, et al. 3D GABA imaging with real-time motion correction, shim update and reacquisition of adiabatic spiral MRSI. Neuroimage. 2014;103:290-302.

[15] Porter DA, Heidemann RM. High resolution diffusion-weighted imaging using readout-segmented echo-planar imaging, parallel imaging and a two-dimensional navigator-based reacquisition. Magn Reson Med. 2009;62:468-475.

[16] Benner T, Van Der Kouwe AJW, Sorensen AG. Diffusion imaging with prospective motion correction and reacquisition. Magn Reson Med. 2011;66:154-167.

[17] Lemieux L, Salek-Haddadi A, Lund TE, Laufs H, Carmichael D. Modelling large motion events in fMRI studies of patients with epilepsy. Magn Reson Imaging. 2007;25:894-901.

[18] Weiskopf N, Suckling J, Williams G, et al. Quantitative multiparameter mapping of R1, PD*, MT, and R2* at 3T: A multicenter validation. Front Neurosci. 2013;7:95.

[19] Callaghan MF, Freund P, Draganski B, et al. Widespread agerelated differences in the human brain microstructure revealed by quantitative magnetic resonance imaging. Neurobiol Aging. 2014;35:1862-1872.

[20] Nöth U, Volz S, Hattingen E, Deichmann R. An improved method for retrospective motion correction in quantitative $\mathrm{T} 2 *$ mapping. Neuroimage. 2014;92:106-119.

[21] Magerkurth J, Volz S, Wagner M, et al. Quantitative $T^{* 2}$ mapping based on multi-slice multiple gradient echo flash imaging: Retrospective correction for subject motion effects. Magn Reson Med. 2011;66:989-997.

[22] Stuber C, Morawski M, Schafer A, et al. Myelin and iron concentration in the human brain: a quantitative study of MRI contrast. Neuroimage. 2014;93:95-106.

[23] Langkammer C, Krebs N, Goessler W, et al. Quantitative MR Imaging of brain iron: a postmortem validation study. Radiology. 2010;257:455-462.

[24] Cohen-Adad J, Polimeni JR, Helmer KG, et al. $\mathrm{T}_{2}^{*}$ mapping and $\mathrm{B}_{0}$ orientation-dependence at $7 \mathrm{~T}$ reveal cyto- and myeloarchitecture organization of the human cortex. Neuroimage. 2012;60:1006-1014.

[25] Ashburner J, Friston KJ. Unified segmentation. Neuroimage. 2005;26:839-851.

[26] Lutti A, Dick F, Sereno MI, Weiskopf N. Using high-resolution quantitative mapping of R1 as an index of cortical myelination. Neuroimage. 2014;93:176-188.

[27] Lutti A, Hutton C, Finsterbusch J, Helms G, Weiskopf N. Optimization and validation of methods for mapping of the radiofrequency transmit field at 3T. Magn Reson Med. 2010;64:229-238.

[28] Lutti A, Stadler J, Josephs O, et al. Robust and fast whole brain mapping of the RF transmit field B1 at 7T. PLoS One. 2012;7: e32379.

[29] Helms G, Dathe H, Kallenberg K, Dechent P. High-resolution maps of magnetization transfer with inherent correction for RF inhomogeneity and T1 relaxation obtained from 3D FLASH MRI. Magn Reson Med. 2008;60:1396-1407.

[30] Bammer R, Aksoy M, Liu C. Augmented generalized SENSE reconstruction to correct for rigid body motion. Magn Reson Med. 2007;57:90-102. 
[31] Banerjee S, Beatty PJ, Zhang JZ, Shankaranarayanan A. Parallel and partial Fourier imaging with prospective motion correction. Magn Reson Med. 2013;69:421-433.

[32] Bailes DR, Gilderdale DJ, Bydder GM, Collins AG, Firmin DN. Respiratory ordered phase encoding (ROPE): a method for reducing respiratory motion artefacts in MR imaging. J Comput Assist Tomogr. 1985;9:835-838.

[33] Cho MH, Kim WS, Cho ZH. CSF flow artifact reduction using cardiac cycle ordered phase-encoding method. Magn Reson Imaging. 1990;8:395-405.

[34] Frost R, Miller KL, Tijssen RHN, Porter DA, Jezzard P. 3D multi-slab diffusion-weighted readout-segmented EPI with realtime cardiac-reordered k-space acquisition. Magn Reson Med. 2014;72:1565-1579.

[35] Pannetier NA, Stavrinos T, Ng P, et al. Quantitative framework for prospective motion correction evaluation. Magn Reson Med. 2016;75:810-816.

[36] Cohen-Adad J. What can we learn from T2* maps of the cortex? Neuroimage. 2014;93:189-200.

[37] Yablonskiy DA, Sukstanskii AL, Luo J, Wang X. Voxel spread function method for correction of magnetic field inhomogeneity effects in quantitative gradient-echo-based MRI. Magn Reson Med. 2013;70:1283-1292.

\section{SUPPORTING INFORMATION}

Additional supporting information may be found in the online version of this article.

FIGURE S1. Histogram of $\mathrm{S}$ values in compliant population. This distribution approximately followed a log-normal distribution (dashed line) with a mean and standard deviation parameters $\mu=-0.083$ and $\sigma=0.568$.

FIGURE S2. Simulation of head motion in the spatially varying sensitivity profiles of the 64 and 20 channel head coils. The simulation was run on the 8 motion time courses that led to large deviations from the linear behaviour shown in Figure 4A. A) Experimental and simulated $\mathrm{SDR}_{2}^{*}$; values. The corresponding $\mathrm{SDR}_{2}^{*}$ obtained experimentally under the no-motion condition are shown for reference. B) Example of acquired $\mathrm{R}_{2}^{*}$ map and corresponding simulated $\mathrm{R}_{2}^{*}$ maps with the 64 and 20 channel profiles.

FIGURE S3. Relationship between image degradation $\left(\mathrm{SDR}_{2}^{*}\right)$ and motion history $(\overline{\Delta M e w})$ calculated before (solid circles) and after (empty circles) shifting the head speed estimates by two points relative to the weights. Results are shown for experiment 2 with the 64 and 20 channel head coils.

FIGURE S4. Relationship between image degradation $\left(\mathrm{SDR}_{2}^{*}\right)$ and motion history $(\overline{\Delta M e w})$ calculated from weights averaged across a reference datasets (solid circles) and the individual weights of the participants to the motion experiment (empty circles). Results are shown for experiment 1 (A) and experiment 2 with the 64 and 20 channel head coils.

FIGURE S5. Relationship between amplitude of the weights and distance to the kspace centre (A). Dependence of image degradation $\left(\mathrm{SDR}_{2}^{*}\right)$ on motion history estimates $\overline{\Delta M e w}$ calculated from the weights obtained from this relationship (solid circles) and the individual weights of the participants to the motion experiment (empty circles). Figure (B) shows results from experiment 1 and figures (C) and (D) from experiment 2 (64 and 20 channel head coils respectively).

How to cite this article: Castella R, Arn L, Dupuis E, Callaghan MF, Draganski B, Lutti A. Controlling motion artefact levels in MR images by suspending data acquisition during periods of head motion. Magn Reson Med. 2018;00:1-12. https://doi.org/10.1002/ mrm.27214 